\title{
Bayesian Testing, Variable Selection and Model Averaging in Linear Models using R with Bayes VarSel
}

by Gonzalo Garcia-Donato and Anabel Forte

\begin{abstract}
In this paper, objective Bayesian methods for hypothesis testing and variable selection in linear models are considered. The focus is on Bayes VarSel, an R package that computes posterior probabilities of hypotheses/models and provides a suite of tools to properly summarize the results. We introduce the usage of specific functions to compute several types of model averaging estimations and predictions weighted by posterior probabilities. BayesVarSel contains exact algorithms to perform fast computations in problems of small to moderate size and heuristic sampling methods to solve large problems. We illustrate the functionalities of the package with several data examples.
\end{abstract}

\section{An illustrated overview of Bayes VarSel}

Testing and variable selection problems are taught in almost any introductory statistical course. In this first section we assume such background to present the essence of the Bayesian approach and the basic usage of Bayes VarSel (Garcia-Donato and Forte, 2015) with hardly any mathematical formulas. Our motivating idea in this first section is mainly to present the appeal of the Bayesian answers to a very broad spectrum of applied researchers. This introductory section concludes with a discussion about connections with potentially related $\mathrm{R}$ packages.

The remaining six sections are organized as follows. In the second section, on page 158, the problem is presented and the notation needed is introduced jointly with the basics of the Bayesian methodology. Then, two sections follow with explanations of the details concerning the obtention of posterior probabilities in hypothesis testing and variable selection problems, respectively, in Bayes VarSel. In a later section, on page 163, several tools to describe the posterior distribution are explained, while the section on page 166 is devoted to model averaging techniques. The paper concludes with a section with plans for the future of the BayesVarSel project. This paper is supplemented with an appendix, with formulas for the most delicate ingredient in the underlying problem in BayesVarSel, namely the prior distributions for parameters within each model.

The version of Bayes VarSel presented here is 1.8.0.

\section{Testing}

In testing problems, several competing hypotheses, $H_{i}$, about a phenomenon of interest are postulated. The role of statistics is to provide summaries about the evidence in favor (or against) the hypotheses once the data, $y$, have been observed. There are many important statistical problems with roots in testing like model selection (or model choice) and model averaging.

The formal Bayesian response to testing problems is based on the posterior probabilities of the hypotheses that summarize, in a fully understandable way, all the available information. In the R package Bayes VarSel a number of popular objective priors (in the sense explained in Berger, 2006) are available. Any of these priors have the great appeal of being fully automatic for users. data:

For illustrative purposes consider the nutrition problem in Lee (1997), page 143, with

$>$ weight.gains <- $c(134,146,104,119,124,161,107,83,113,129,97,123$,

$+70,118,101,85,107,132,94)$

There it is tested, based on the sample of 19 weight gains (expressed in grams) of rats, whether there is a difference between the population means of the group with a high proteinic diet (the first 12) or the control group (the rest): 
$>$ diet <- as.factor $(c(\operatorname{rep}(1,12), \operatorname{rep}(0,7)))$

$>$ rats <- data.frame (weight.gains = weight.gains, diet $=$ diet $)$

This problem (usually known as the two-samples $t$-test) is normally written as $H_{0}: \mu_{1}=\mu_{2}$ versus $H_{1}: \mu_{1} \neq \mu_{2}$, where it is assumed that the weight gains are normally distributed with an unknown (but common) standard deviation. The formulas that define each of the models under the postulated hypotheses are in $\mathrm{R}$ language

$>$ MO <- weight.gains $\sim 1$

$>$ M1 <- weight.gains diet

The function to perform Bayesian tests in BayesVarSel is Btest which has an intuitive and simple syntax (see the section on page 160 for a detailed description). In this example

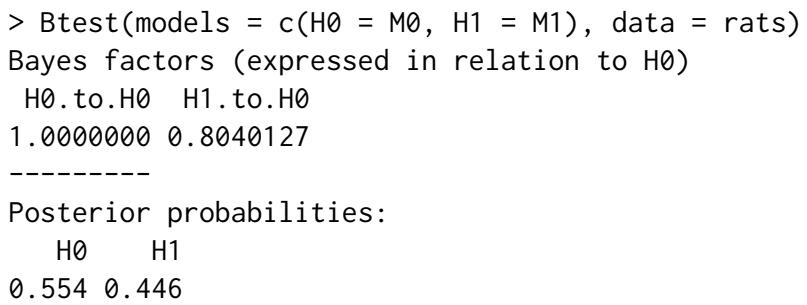

From these results, we clearly conclude that both hypotheses are similarly supported by the data. Hence there is no evidence that the diet has any impact on the average weight.

A useful guide to interpret Bayes factors and probabilities, follows from the categories proposed by Kass and Raftery (1995), reproduced in Table 1. For the problem above, none of the hypotheses is worth more than a bare mention.

\begin{tabular}{lll}
$B_{10}$ & Probability & Evidence against the null \\
\hline 1 to 3 & 0.5 to 0.75 & Not worth more than a bare mention \\
3 to 20 & 0.75 to 0.95 & Substantial \\
20 to 150 & 0.95 to 0.99 & Strong \\
$>150$ & $>0.99$ & Decisive
\end{tabular}

Table 1: Interpretation of Bayes factors from Kass and Raftery (1995) ( $B_{10}$ stands for the Bayes factor of $H_{1}$ to the null hypothesis $H_{0}$ ). The column probability is obtained assuming that both hypotheses are equally likely a priori.

Another illustrative example concerns the classic dataset savings in Belsley et al. (2005) considered by Faraway (2002), page 29 and distributed under the package faraway (Faraway, 2016).

$>$ data("savings", package = "faraway")

This dataset contains macroeconomic data on 50 different countries during 1960-1970 and the question posed is to elucidate if dpi (per-capita disposable income in U.S), ddpi (percent rate of change in per capita disposable income), population under (over) 15 (75) pop15 (pop75) are all explanatory variables for sr, the aggregate personal saving divided by disposable income which is assumed to follow a normal distribution. This can be written as a testing problem about the regression coefficients associated with the variables with hypotheses

$$
H_{0}: \beta_{d p i}=\beta_{d d p i}=\beta_{p o p 15}=\beta_{p o p 75}=0,
$$

versus the alternative, say $H_{1}$, that all predictors are needed. The competing models can be defined as

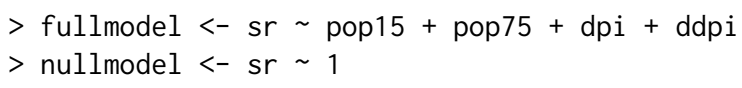




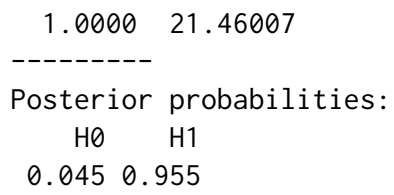

Now, the conclusion is that there is strong evidence favoring $H_{1}$, the hypothesis that all considered predictors explain the response sr.

Of course, more hypotheses can be tested at the same time. For instance, a simplified version of $H_{1}$ that does not include pop15 is

$$
H_{2}: \beta_{d p i}=\beta_{d d p i}=\beta_{p o p 75}=0,
$$

that can be included in the analysis as

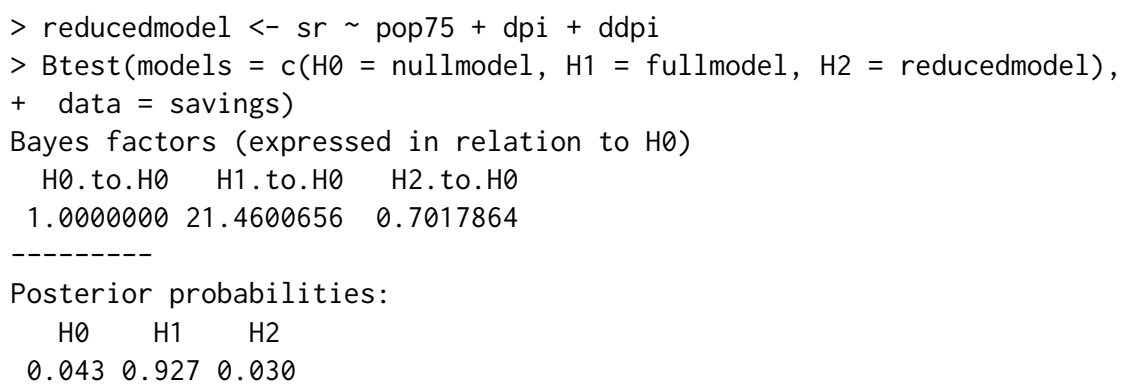

Obviously, as more hypotheses are considered, the usefulness of Table 1 to interpret posterior probabilities reduces since it was conceived for two hypotheses. Nevertheless, in this case, $H_{1}$ is clearly supported by the data as the best explanation for the experiment among those considered.

This scenario can be extended to check which subset of the four covariates is the most suitable one to explain sr. In general, the problem of selecting the best subset of covariates from a group of potential ones is better known as variable selection.

\section{Variable selection}

Variable selection is a multiple testing problem where each hypothesis proposes a possible subset of the $p$ potential explanatory variables initially considered. Notice that there are $2^{p}$ hypotheses, including the simplest one (the null hypothesis) stating that none of the variables should be used.

A variable selection approach to the economic example above with $p=4$ has 16 hypotheses and can be solved using the Btest function. Nevertheless, BayesVarSel has specific facilities to handle the specificities of variable selection problems. A main function for variable selection is Bvs, fully described in the section on page 161. It has a simple syntax inspired by the well-known $1 \mathrm{~m}$ function. The variable selection problem in this economic example can be solved by executing:

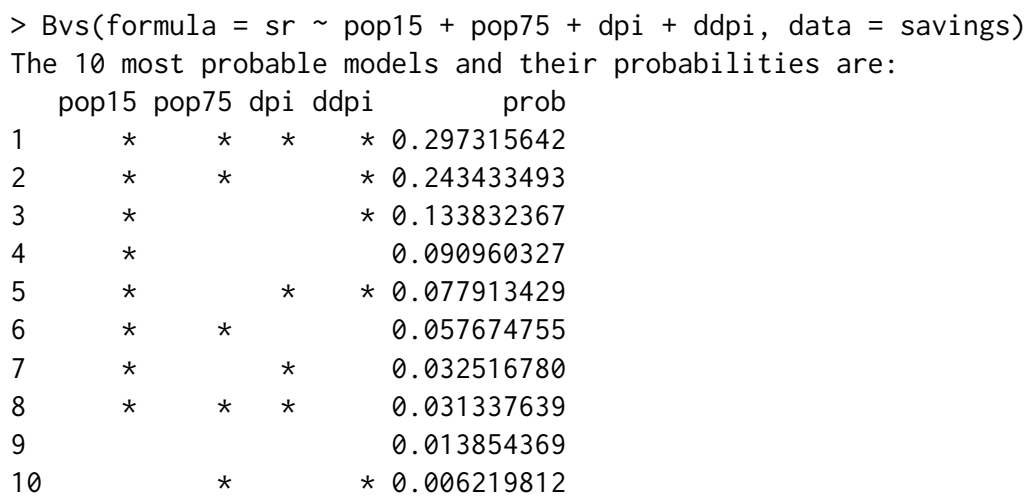

With a first look at these results, we can see that the most probable model is the model with all covariates (probability 0.30 ), which is closely followed by the one without dpi with a 
posterior probability of 0.24 . Note that the results are sensitive to the choice of parameter priors (see the appendix for more details on the priors).

As we will see later, a variable selection exercise generates a lot of valuable information of which the above printed information is only a very reduced summary. This additional information can be accessed with specific methods that explore the characteristics of objects of the type created by Bvs.

\section{Related packages}

In a recent study, Forte et al. (2018) analyse the differences among available R packages that, according to either title and/or description, perform common variable selection (and related) tasks. The main underlying motivation in that study was to clarify the commonalities/differences of the different packages available sharing a common theoretical framework (Bayesian variable selection with a particular type of priors). Five packages turned out to have these characteristics: BayesFactor (Morey et al., 2015); BayesVarSel; BMS (Zeugner and Feldkircher, 2015); mombf (Rossell et al., 2014) and BAS (Clyde, 2017). These were further compared taking into account relevant aspects like flexibility on prior specification, types of summaries provided, and even computational skills. The main conclusion was that, despite the connections, there are important differences (e.g., on prior specifications or implemented summaries) that will dictate the choice of package (for more details see Forte et al., 2018).

Another package worth mentioning is BMA (Raftery et al., 2015). This is one of the most downloaded packages for variable selection in CRAN. BMA performs variable selection based on the (BIC) Bayesian Information Criterion (which was developed as an asymptotic approximation to Bayes factors).

\section{Basic formulae}

The problems considered in Bayes VarSel concern Gaussian linear models. Consider a response variable $y$, size $n$, assumed to follow the linear model (the subindex $F$ refers to the full model)

$$
M_{F}: y=X_{0} \boldsymbol{\alpha}+\boldsymbol{X} \boldsymbol{\beta}+\varepsilon, \varepsilon \sim N_{n}\left(\mathbf{0}, \sigma^{2} \mathbf{I}_{n}\right),
$$

where the matrices $\boldsymbol{X}_{0}: n \times p_{0}, \boldsymbol{X}: n \times p$ and the regression vector coefficients are of conformable dimensions. Suppose you want to test $H_{0}: \boldsymbol{\beta}=\mathbf{0}$ versus $H_{F}: \boldsymbol{\beta} \neq \mathbf{0}$, that is, to decide whether the regressors in $X$ actually explain the response. This problem is equivalent to the model choice (or model selection) problem with competing models $M_{F}$ and

$$
M_{0}: y=X_{0} \boldsymbol{\alpha}+\varepsilon, \varepsilon \sim N_{n}\left(\mathbf{0}, \sigma^{2} I_{n}\right),
$$

and we will refer to models or hypotheses indistinctly.

Posterior probabilities are based on the Bayes factors (see Kass and Raftery, 1995), a measure of evidence provided by BayesVarSel when solving testing problems. The Bayes factor of $H_{F}$ to $H_{0}$ is

$$
B_{F 0}=\frac{m_{F}(\boldsymbol{y})}{m_{0}(\boldsymbol{y})},
$$

where $m_{F}$ is the integrated likelihood or prior predictive marginal for the full model:

$$
m_{F}(\boldsymbol{y})=\int M_{F}(\boldsymbol{y} \mid \boldsymbol{\alpha}, \boldsymbol{\beta}, \sigma) \pi_{F}(\boldsymbol{\alpha}, \boldsymbol{\beta}, \sigma) d \boldsymbol{\beta} d \boldsymbol{\alpha} d \sigma,
$$

and, similarly:

$$
m_{0}(\boldsymbol{y})=\int M_{0}(\boldsymbol{y} \mid \boldsymbol{\alpha}, \sigma) \pi_{0}(\boldsymbol{\alpha}, \sigma) d \boldsymbol{\alpha} d \sigma .
$$

Above, $\pi_{0}$ and $\pi_{F}$ are the prior distributions for the parameters within each model. The assignment of such priors (which we call model selection priors) is quite a delicate issue (see Berger and Pericchi, 2001) and has inspired many important contributions in the literature, particularly from an objective point of view. Of these, the package BayesVarSel allows using 
many of the most important proposals, which are fully detailed in the appendix. The prior implemented by default is the robust prior by Bayarri et al. (2012), as it can be considered optimal in many senses and is based on a foundational basis.

Posterior probabilities can be obtained as

$$
\operatorname{Pr}\left(H_{F} \mid \boldsymbol{y}\right)=\frac{B_{F 0} \operatorname{Pr}\left(H_{F}\right)}{\left(\operatorname{Pr}\left(H_{0}\right)+B_{F 0} \operatorname{Pr}\left(H_{F}\right)\right)}, \operatorname{Pr}\left(H_{0} \mid \boldsymbol{y}\right)=1-\operatorname{Pr}\left(H_{F} \mid \boldsymbol{y}\right),
$$

where $\operatorname{Pr}\left(H_{F}\right)$ is the probability, a priori, that hypothesis $H_{F}$ is true.

Similar formulas can be obtained when more than two hypotheses, say $H_{1}, \ldots, H_{N}$, are tested. In this case

$$
\operatorname{Pr}\left(H_{i} \mid \boldsymbol{y}\right)=\frac{B_{i 0}(\boldsymbol{y}) \operatorname{Pr}\left(H_{i}\right)}{\sum_{j=1}^{N} B_{j 0}(\boldsymbol{y}) \operatorname{Pr}\left(H_{j}\right)}, i=1, \ldots, N,
$$

which is the posterior distribution over the model space (the set that contains all competing models). For simplicitly, the formula in (3) has been expressed, without any loss of generality, using Bayes factors to the null model but the same results would be obtained by fixing any other model. The default definition for $\operatorname{Pr}\left(H_{i}\right)$ in testing problems is to use a constant prior, which assigns the same probability to all models, that is, $\operatorname{Pr}\left(H_{i}\right)=1 / \mathrm{N}$.

For instance, within the model

$$
M_{3}: y=\alpha \mathbf{1}_{n}+\beta_{1} x_{1}+\beta_{2} x_{2}+\varepsilon,
$$

we cannot test the hypotheses $H_{1}: \beta_{1}=0, \beta_{2} \neq 0, H_{2}: \beta_{1} \neq 0, \beta_{2}=0, H_{3}: \beta_{1} \neq 0, \beta_{2} \neq 0$ since neither $M_{1}$ (the model defined by $H_{1}$ ) nor $M_{2}$ are nested in the rest. Nevertheless, it is perfectly possible to test the problem with the four hypotheses $H_{1}, H_{2}, H_{3}$ (as just defined) plus $H_{0}: \beta_{1}=0, \beta_{2}=0$, but of course $H_{0}$ must be, a priori, a plausible hypothesis. In this last case $H_{0}$ would take the role of null model.

Hypotheses do not have to be necessarily of the type $\beta=0$ and, if testable (see Ravishanker and Dey, 2002, for a proper definition), any linear combination of the type $C^{t} \boldsymbol{\beta}=\mathbf{0}$ can be considered a hypothesis. For instance one can be interested in testing $\beta_{1}+\beta_{2}=0$. In Bayarri and García-Donato (2007) it was formally shown that these hypotheses can be, through reparameterizations, reduced to hypotheses like $\beta=0$. In next section we show examples of how to solve these testing problems in BayesVarSel .

Variable selection is a multiple testing problem but is traditionally presented with convenient specific notation that uses a $p$ dimensional binary vector $\gamma=\left(\gamma_{1}, \ldots, \gamma_{p}\right)$ to identify the models. Consider the full model in (1), and suppose that $\boldsymbol{X}_{0}$ contains fixed covariates that are believed to be sure in the true model (by default $X_{0}=\mathbf{1}_{n}$ that would make the intercept present in all the models). Then each $\gamma \in\{0,1\}^{p}$ defines a hypothesis $H_{\gamma}$ stating which $\beta^{\prime}$ s (those with $\gamma_{i}=0$ ) corresponding to each of the columns in $\boldsymbol{X}$ are zero. Then, the model associated with $H_{\gamma}$ is

$$
M_{\gamma}: \boldsymbol{y}=\boldsymbol{X}_{0} \boldsymbol{\alpha}+\boldsymbol{X}_{\gamma} \boldsymbol{\beta}_{\gamma}+\varepsilon, \varepsilon \sim N_{n}\left(\mathbf{0}, \sigma^{2} \boldsymbol{I}_{n}\right),
$$

where $X_{\gamma}$ is the matrix with the columns in $X$ corresponding to the ones in $\gamma$. Notice that $\boldsymbol{X}_{\gamma}$ is a $n \times p_{\gamma}$ matrix where $p_{\gamma}$ is the number of 1 's in $\gamma$.

Clearly, in this variable selection problem there are $2^{p}$ hypotheses or models and the null model is (2) corresponding to $\boldsymbol{\gamma}=\mathbf{0}$.

A particularity of variable selection is that it is affected by multiplicity issues. This is because, and specially for moderate to large $p$, the possibility of a model showing spurious evidence is high (just because many hypotheses are considered simultaneously). As concluded in Scott and Berger (2006) multiplicity must be controlled with the prior probabilities $\operatorname{Pr}\left(H_{\gamma}\right)$ and the constant prior does not control for multiplicity. Instead, these authors propose using

$$
\operatorname{Pr}\left(H_{\gamma}\right)=\left((p+1)\left(\begin{array}{c}
p \\
p_{\gamma}
\end{array}\right)\right)^{-1}
$$


The assignment above states that models of the same dimension (the dimension of $M_{\gamma}$ is $p_{\gamma}+p_{0}$ ) should have the same probability which must be inversely proportional to the number of models of that dimension. In the sequel we refer to this prior as the ScottBerger prior.

Both the ScottBerger prior and the Constant prior for $\operatorname{Pr}\left(H_{\gamma}\right)$ are particular cases of the very flexible prior

$$
\operatorname{Pr}\left(M_{\gamma} \mid \theta\right)=\theta^{p_{\gamma}}(1-\theta)^{p-p_{\gamma}},
$$

where the hyperparameter $\theta \in(0,1)$ has the interpretation of the common probability that a given variable is included (independently of all others).

The Constant prior corresponds to $\theta=1 / 2$ while the ScottBerger to $\theta \sim \operatorname{Unif}(0,1)$. Ley and Steel (2009) study priors for $\theta$ of the type

$$
\theta \sim \operatorname{Beta}(\theta \mid 1, b)
$$

They argue that, on many occasions the user has, a priori, some information regarding the number of covariates (among the $p$ initially considered) that are expected to explain the response, say $w^{\star}$. As they explain, this information can be translated into the analysis assigning in (7) $b=\left(p-w^{\star}\right) / w^{\star}$. The resulting prior specification has the property that the expected number of covariates is precisely $w^{\star}$. out $\theta$ )

Straightforward algebra shows that assuming (7) into (6) is equivalent to (integrating

$$
\operatorname{Pr}\left(M_{\gamma} \mid b\right) \propto \Gamma\left(p_{\gamma}+1\right) \Gamma\left(p-p_{\gamma}+b\right) .
$$
prior.

The prior over the model space implemented by default in Bayes VarSel is the ScottBerger

\section{Hypothesis testing with Bayes VarSel}

Tests are solved in Bayes VarSel with Btest which, in its default usage, only depends on two arguments: models a named list of formula-type objects defining the models compared and data the data frame with the data.

The prior probabilities assigned to the hypotheses is constant, that is, $\operatorname{Pr}\left(H_{i}\right)=1 / \mathrm{N}$. This default behavior can be modified specifying prior.models = "User" jointly with the argument priorprobs that must contain a named list (with names as specified in the main argument models) with the prior probabilities to be used for each hypotheses.

Suppose that in the last example of first subsection, we establish a priori that the simpler model is twice as likely as the other two. This can be specified as:

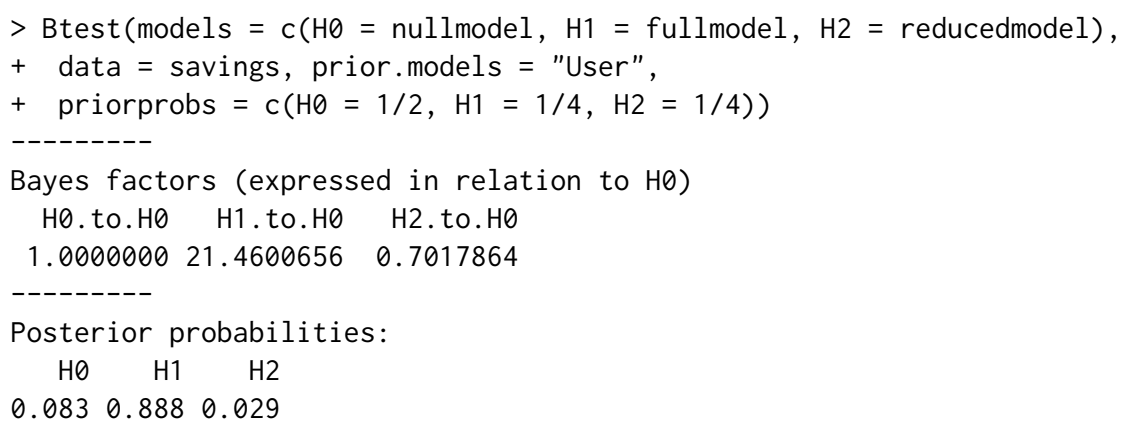

Notice that the Bayes factor remains the same, and the change is in posterior probabilities.

Btest tries to identify the null model (i.e. the model nested in all the others) using the names of the variables. If such a model does not exist, the execution of the function stops with an error message. Nevertheless, there are important situations where the simplest (null) hypothesis is defined through linear restrictions (sometimes known as 'testing a subspace') making it very difficult to determine its existence just using the names. In this situation, the user must provide the name of the simplest model in the argument null.model.

To illustrate this case, consider for instance the extension of the savings example in Faraway (2002), page 32 where $H_{\text {eqp }}: \beta_{\text {pop } 15}=\beta_{\text {pop } 75}$ is tested against the full alternative. 
This null hypothesis states that the effect on personal savings, sr, of both segments of populations is similar. The model under $H_{\text {eqp }}$ can be specified as:

$>$ equalpopmodel <- sr I (pop15 + pop75) + dpi + ddpi

but the command

$>$ Btest $($ models $=\mathrm{c}($ Heqp $=$ equalpopmodel, $\mathrm{H} 1=$ fullmodel $)$, data $=$ savings $)$

produces an error, although it is clear that $H_{\text {eqp }}$ is nested in $H_{1}$. To overcome this error, the user must make the name of the null model explicit. In our example:

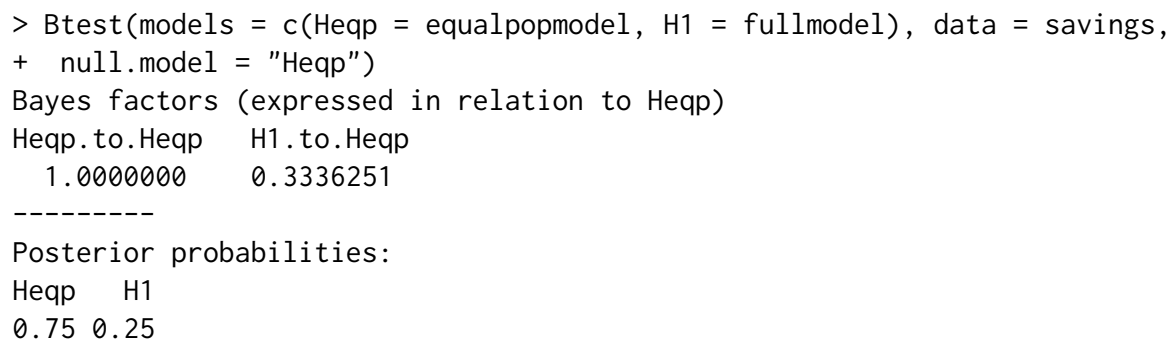

Still, the code would produce an error if it detects that the model defined as the null model does not have the largest sum of squared errors or if it is not of a smaller dimension than all the others.

\section{Variable selection with Bayes VarSel}

The number of entertained hypotheses in a variable selection problem, $2^{p}$, can range from a few to an extremely large number. This makes it necessary to program specific tools to solve the multiple testing problem in variable selection problems. BayesVarSel provides two different functions for variable selection

- Bvs performs exhaustive enumeration of hypotheses and hence the size of problems must be small or moderate (say $p \leq 25$ ),

- GibbsBvs simulates from the posterior distribution over the model space using a Gibbs sampling scheme (intended to be used for large problems, with $p>25$ ).

Except for a few arguments that are specific to the algorithm implemented (e.g., the number of cores in Bvs or the number of simulations in GibbsBvs) the usage of the two functions is very similar. We describe the common use in the first of the following subsections and the function-specific arguments in the second.

Bvs and GibbsBvs return objects of class "Bvs" which are a list with relevant information about the posterior distribution. For these objects, BayesVarSel provides a number of functions, based on the tradition of model selection methods, to summarize the corresponding posterior distribution (e.g., what is the hypothesis most probable a posteriori) and for its posterior usage (e.g., to obtain predictions or model averaged estimates). These capabilities are described in next two sections.

For illustrative purposes we use the following datasets:

UScrime data. The US crime data set was first studied by Ehrlich (1973) and is available from the package MASS (Venables and Ripley, 2002). This data set has a total of $n=47$ observations (corresponding to states in the US) of $p=15$ potential covariates aimed at explaining the rate of crimes in a particular category per head of population (labelled $y$ in the data).

SDM data. This dataset has a total of $p=67$ potential drivers for the annual GDP growth per capita between 1960 and 1996 for $n=88$ countries (response variable labelled $y$ in the data). This data set was initially considered by Sala-I-Martin et al. (2004) and revisited by Ley and Steel (2007). 


\section{Common arguments}

The customary arguments in Bvs and GibbsBvs are formula, with a definition of the most complex model considered (the full model in (1)) and data (a data frame with the data). The default execution setting corresponds to a problem where the null model (2) contains just the intercept (i.e. $\boldsymbol{X}_{0}=\mathbf{1}_{n}$ ) and prior probabilities for models are defined as in (5).

A different null model can be specified with the optional argument null.model, that must take the value of the formula of the the null model (a model that should be nested in the full model).

Suppose for example that in the UScrime dataset and apart from the constant, theory suggests that the covariate Ed must be used to explain the dependent variable. To consider these conditions we execute the command

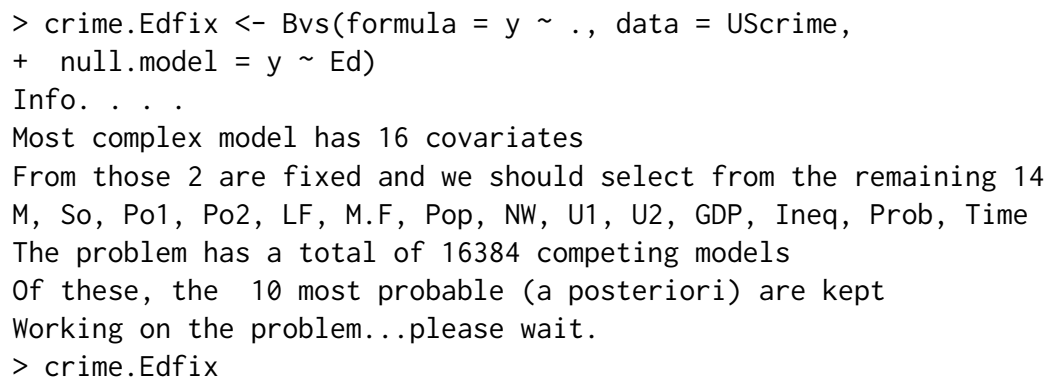

During the execution (which takes about 0.22 seconds in a standard laptop) the function informs which variables take part of the selection process. The number of these defines $p$ which in this problem is $p=14$ (and the model space has $2^{14}$ models). In what follows, and unless otherwise stated we do not reproduce this informative output to save space.

The assignment of priors probabilities, $\operatorname{Pr}\left(H_{i}\right)$, is regulated with prior . models, an argument that by default takes the value "ScottBerger"' corresponding to the proposal in (5). Other options for this argument are "'Constant"', which stands for $\operatorname{Pr}\left(H_{i}\right)=1 / 2^{p}$, and the more flexible value, '"User"', under which the user must specify the prior probabilities with the extra argument priorprobs.

The argument priorprobs is a $p+1$ numeric vector, which in its $i$-th position defines the probability of a model of dimension $p_{0}+i-1$ (these probabilities can be specified except for the normalizing constant).

Suppose that in the UScrime dataset with null model just the intercept, we want to specify the prior in eq (6) with $\theta=1 / 4$, this can be done as (notice that here $p=15$ )

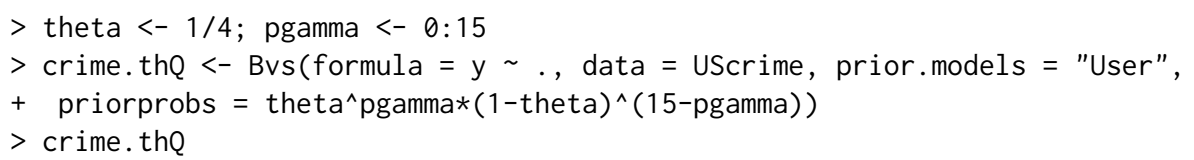

In variable selection problems it is quite standard to have the situation where the number of covariates is large (say larger than 30) preventing the exhaustive enumeration of all the competing models. The SDM dataset is an example of this situation with $p=67$. In these contexts, the posterior distribution can be explored using the function GibbsBvs. To illustrate the elicitation of prior probabilities as well, suppose that the number of expected covariates to be present in the true model is $w^{\star}=7$. This situation is considered in Ley and Steel (2009) and can be implemented as (see (8))

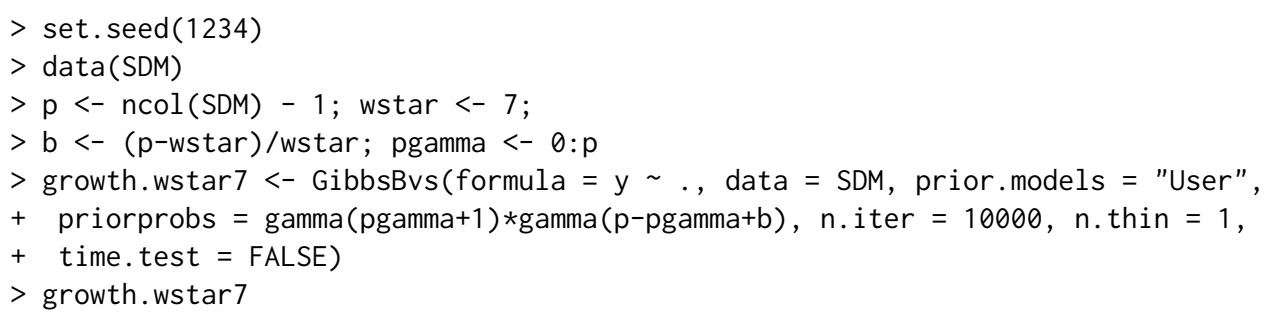

The above code took 18 seconds to run.

One last common argument to Bvs and GibbsBvs is time. test. If it is set to TRUE and the problem is of moderate size ( $p \geq 18$ in Bvs and $p \geq 21$ in GibbsBvs), an estimation of 
computational time is calculated and the user is asked about the possibility of not executing the command.

\section{Specific arguments}

In Bvs The algorithm implemented in Bvs is exact in the sense that the information collected about the posterior distribution takes into account all competing models as these are all computed. The logical parameter parallel, if set to TRUE, distributes the task among the number of processors specified in the argument $\mathrm{n}$. nodes. By default the parameter parallel is set to FALSE. To save computational time and memory it is quite appropriate to keep only a moderate number of the best (most probable a posteriori) models. This number can be specified with the argument $n$. keep which must be an integer number between 1 (only the most probable model is kept) and $2^{p}$ (a full ordering of models is kept) (or $2^{p}$ divided by $\mathrm{n}$. nodes in the case of parallel computation). The default value for $n$. keep is 10 .

The argument $n$. keep is not of great importance to analyze the posterior distribution over the model space. Nevertheless, it has a more relevant effect if model averaging estimates or predictions are to be obtained (see the corresponding section on page 166) since, as BayesVarSel is designed, only the $\mathrm{n}$. keep retained models are used for these tasks.

In GibbsBvs The algorithm in GibbsBvs samples models from the posterior over the model space and this is done using a simple (yet very efficient) Gibbs sampling scheme introduced in George and McCulloch (1997), later studied in Garcia-Donato and Martinez-Beneito (2013) in the context of large model spaces. The type of default arguments that can be specified in GibbsBvs are typical in any Monte Carlo Markov Chain scheme (as usual the default values are given in the assignment)

- init.model = "Full" The model at which the simulation process starts. Options include "Null"' (the model only with the covariates specified in null .model), '"Full"' (the model defined by formula), and '"Random"' (a randomly selected model) a vector with $p$ zeros and ones defining a model.

- $\mathrm{n}$. burnin $=500$ Length of burn in, i.e. the number of iterations to discard at the start of the simulation process.

- $\mathrm{n}$. iter $=10000$ The total number of iterations performed after the burn in process.

- $\mathrm{n}$. thin $=1$ Thinning rate that must be a positive integer. Set $\mathrm{n}$. thin $>1$ to save memory and computation time if $\mathrm{n}$. i ter is large.

- seed $=\operatorname{runif}(1,0,16091956)$ A seed to initialize the random number generator.

Notice that the number of total iterations is $n$. burnin+n. iter but the number of models that are used to collect information from the posterior is, approximately, n. iter $/ \mathrm{n}$. thin.

\section{Summaries of the posterior distribution}

In this section we describe the tools implemented in BayesVarSel conceived to summarize (in the tradition of the relevant model selection literature) the posterior distribution over the model space. In R, this corresponds to describing methods to explore the content of objects of class "Bvs".

Printing a "Bvs" object created with Bvs shows the best 10 models with their associated probability (see examples in first section). If the object was built with GibbsBvs the 10 most probable a posteriori models among the visited ones are shown. Though, in this case, posterior probabilities of models are not provided, as these are unknown.

The rest of the summaries are very similar, independent of the routine used to create it, but recall that if the object was obtained with Bvs (likely because $p$ is small or moderate) the given measures here explained are exact. If instead GibbsBvs was used, the reported measures are approximations of the exact ones (that likely cannot be computed due to 
the huge size of the model space). In BayesVarSel these approximations are based on the frequency of visits of $M_{\gamma}$ as an estimator since, as studied in Garcia-Donato and MartinezBeneito (2013), these provide quite accurate summaries.

The HPM is returned when an object of class "Bvs" is summarized (via summary) jointly with the inclusion probabilities for each competing variable, $\operatorname{Pr}\left(x_{i} \mid \boldsymbol{y}\right)$. These are the sum of the posterior probabilities of models containing that covariate and provide evidence about the individual importance of each explanatory variable. The model defined by those variables with an inclusion probability greater than 0.5 is called a Median Probability Model (MPM), which is also included in the summary. Barbieri and Berger (2004) show that, under general conditions, if a single model has to be utilized with predictive purposes, the MPM is optimal.

For instance, if we summarize the object crime. $\operatorname{Edfix}^{1}$ of the fourth section, we obtain:

$>\operatorname{summary}($ crime.Edfix)

Inclusion Probabilities:

Incl.prob. HPM MPM

M 0.6806 *

So $\quad 0.2386$

Po1 0.8489 * *

Po2 0.3663

LF $\quad 0.2209$

M.F $\quad 0.3184$

Pop $\quad 0.2652$

NW $\quad 0.2268$

U1 $\quad 0.2935$

U2 $\quad 0.4765$

GDP $\quad 0.3204$

Ineq $\quad 0.9924$

Prob $\quad 0.6174$

Time $\quad 0.2434$

Inclusion probabilities implicitly answer a testing problem with two hypotheses, namely if the variable should or should not be included. This is made sensible (and practical) using the categorization in Table 1 to interpret them. With this guide, the importance of Ineq is decisive followed by Po1, M and Prob with a substantial evidence in favour of their relevance in explaining the response.

Graphical summaries and jointness The main graphical support in BayesVarSel is contained in the S3 function plot ${ }^{2}$ which depends on $x$ (an object of class "Bvs") and the argument option specifying the type of plot to be produced:

- option=" joint" A matrix plot with the joint inclusion probabilities, $\operatorname{Pr}\left(x_{i}, x_{j} \mid \boldsymbol{y}\right)$ (marginal inclusion probabilities in the diagonal).

- option="conditional" A matrix plot with $\operatorname{Pr}\left(x_{i} \mid x_{j}, y\right)$, the conditional inclusion probabilities (ones in the diagonal).

- option="not" A matrix plot with $\operatorname{Pr}\left(x_{i} \mid\right.$ Not $\left.x_{j}, y\right)$, the conditional inclusion probabilities (zeroes in the diagonal).

- option="dimension" A bar plot representation of the posterior distribution of the dimension of the true model (number of variables, ranging from $p_{0}$ to $p_{0}+p$ ).

- option="trace" A trace plot with the evolution of the inclusion probabilities with the iterations in Gibbs sampling (useful to check convergence; only available for GibbsBvs).

\footnotetext{
${ }^{1}$ Notice that variable Ed is not on the list as it was assumed to be fixed.

${ }^{2}$ Depending on $p$, this function may produce very large plots causing margin-type errors to be thrown if either the device or the active graphical window are too small. In these cases the user must consider ways of enlarging the graphical output recipient.
} 
The first three options above are basic measures describing aspects of the joint effect of two given variables, $x_{i}$ and $x_{j}$, and can be understood as natural extensions of the marginal inclusion probabilities. In Figure 1, we have reproduced the first three plots (from left to right) obtained with the following lines of code:

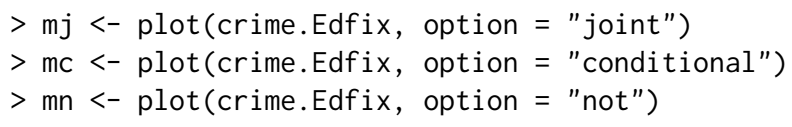

Apart from the plot, these functions return (invisibly) the matrix represented for futher study. For the conditional probabilities (conditional and not) the variables in the row are the conditioning variables (e.g., in $\mathrm{mc}$ above, the position $(i, j)$ is the inclusion probability of variable in $j$-th column conditional on the variable in $i$-th row).

Within these matrices, the most interesting results correspond to variations from the marginal inclusion probabilities (represented in the top of the plots as a separate row for reference). Our experience suggests that the most valuable of these is option="not", as it can reveal key details about the relations between variables in relation with the response. For instance, take that plot in Figure 1 (plot on the left of the second row) and observe that while variable Po2 barely has any effect on y (crime), it becomes relevant if Po1 is removed. This is the probability $\operatorname{Pr}(\operatorname{Po} 2 \mid \operatorname{Not} P o 1, y)$ with value

$>\mathrm{mn}[$ "Not.Po1", "Po2"]

[1] 0.9996444

which, as we observed in the graph, is substantially large compared with the inclusion probability of Po2

$>$ crime.Edfix\$inclprob["Po2"]

Po2

0.3662537

Similarly, we observe that Po1 is of even more importance if Po2 is not considered as a possible explanatory variable. All this implies that, in relation with the dependent variable, both variables contain similar information and one can act as proxy for the other.

We can further investigate this idea of a relationship between two covariates with respect to the response using the jointness measures proposed by Ley and Steel (2007). These are available using a function Jointness that depends on two arguments: $x$, an object of class "Bvs" and covariates, a character vector indicating which pair of covariates we are interested in. The default covariates="All" will output the matrices with the jointness measurement for every pair of covariates. In particular, three jointness measures relative to two covariates are reported by this function: i) the joint inclusion probability, ii) the ratio between the joint inclusion probability and the probability of including at least one of them, and iii) the ratio between the joint inclusion probability and the probability of including one of them alone.

For instance:

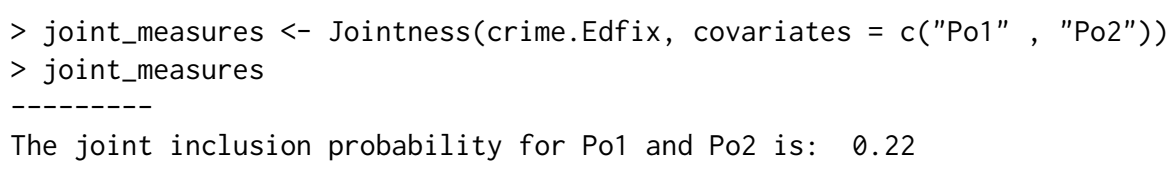

The probability of including both covariates together is 0.27 times the probability of including one of them alone

Alternatively, the single numbers can be accessed using:

$>$ joint_measures\$prob_joint

[1] 0.215181

$>$ joint_measures\$joint_LS1

[1] 0.2151926

$>$ joint_measures\$joint_LS2

[1] 0.274198 

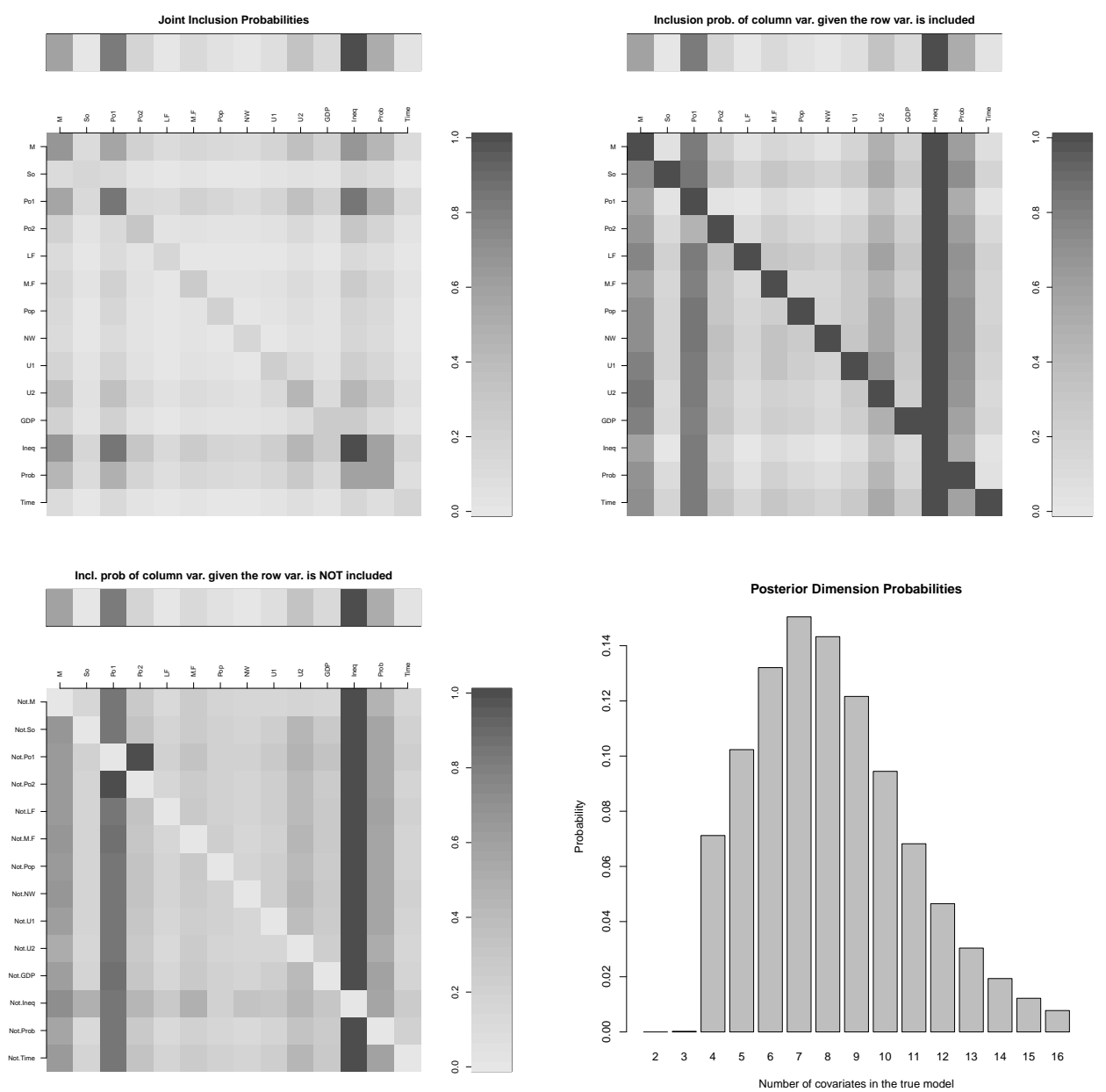

Figure 1: Plots corresponding to the four possible values of the argument option in plot over the object crime. Edfix of section four. From left to right: joint, conditional, not, and dimension.

With these results we must conclude that it is unlikely that both variables, Po1 and Po2, are to be included together in the true model.

Finally, within plot, the assignment option="dimension" produces a plot that speaks about the complexity of the true model in terms of the number of covariates that it contains. The last plot in Figure 1 is the output of executing:

$>\operatorname{plot}($ crime.Edfix, option = "dimension")

From this plot we conclude that the number of covariates is about 7 but with a high variability. The exact values of this posterior distribution are in the component postprobdim of the Bvs object.

The last possibility for the plot method corresponds to option=" trace" which produces a trace plot of the inclusion probabilities. The resulting graph is useful to asses the convergence of the posterior inclusion probabilities when Gibbs sampling was used and we can informally check if the number of iterations is enough. For instance, Figure 2 shows how the posterior inclusion probabilities in this setting stabilize after roughly 8000 iterations.

$>$ plot (growth.wstar7, option $=$ "trace")

\section{Model averaged estimations and predictions}

In a variable selection problem it is explicitly recognized that there is uncertainty regarding which variables make up the true model. Obviously, this uncertainty should be propagated in the inferential process (as opposed to inferences using just one model) to produce more reliable and accurate estimations and predictions. These type of procedures are normally 


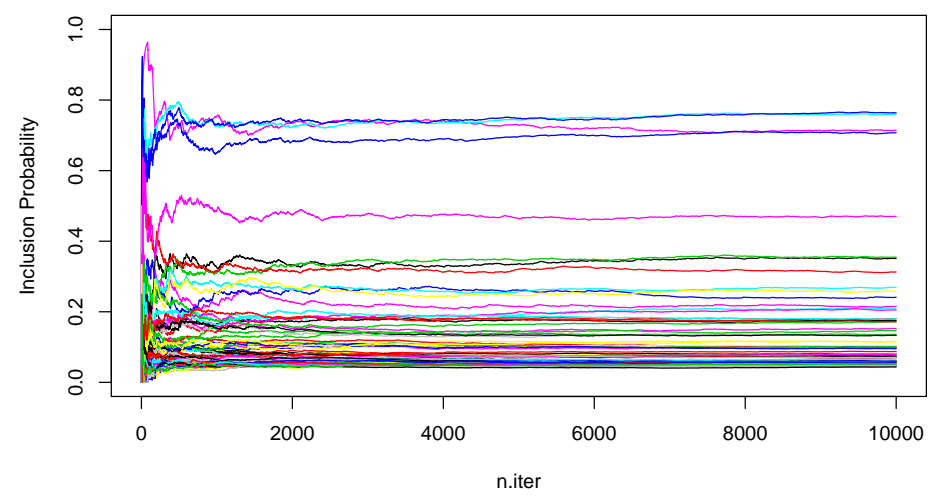

Figure 2: Plot corresponding to the "trace" option in plot over the object growth.wstar7.

called model averaging (Madigan and Raftery, 1994; Raftery, 1995) and are performed once the model selection exercise is performed (that is, the posterior probabilities have been already obtained). In BayesVarSel these inferences can be obtained acting over objects of class "Bvs".

Suppose that $\Lambda$ is a quantity of interest and that under model $M_{\gamma}$ it has a posterior distribution $\pi^{N}\left(\Lambda \mid y, M_{\gamma}\right)$ with respect to certain non-informative prior $\pi_{\gamma}^{N}$. Then, we can average over all entertained models using the posterior probabilities in (3) as weights to obtain

$$
f(\Lambda \mid \boldsymbol{y})=\sum_{\gamma} \pi^{N}\left(\Lambda \mid \boldsymbol{y}, M_{\gamma}\right) \operatorname{Pr}\left(M_{\gamma} \mid \boldsymbol{y}\right) .
$$

In BayesVarSel for $\pi_{\gamma}^{N}$ we use the reference prior developed in Berger and Bernardo (1992) and further studied in Berger et al. (2009). This is an objective prior with very good theoretical properties. The formulas for the posterior distribution with a fixed model are known (Bernardo and Smith, 1994). These priors are different from the model selection priors used to compute the Bayes factors (see the second section), but, as shown in Consonni and Deldossi (2016), the posterior distributions approximately coincide, and so $f(\Lambda \mid \boldsymbol{y})$ basically can be interpreted as the posterior distribution of $\Lambda$.

There are two different quantities, $\Lambda$, that are of main interest in variable selection problems. First is a regression parameter $\beta_{i}$ and second is a future observation $y^{\star}$ associated with known values of the covariates $x^{\star}$. In what follows we refer to each of these problems as (model averaged) estimation and prediction, respectively, to which we devote the next subsections.

\section{Estimation}

Inclusion probabilities $\operatorname{Pr}\left(x_{i} \mid \boldsymbol{y}\right)$ can be roughly interpreted as the probability that $\beta_{i}$ is different from zero. Nevertheless, it does not say anything about the magnitude of the coefficient $\beta_{i}$ nor anything about its sign.

Such type of information can be obtained from the distribution in (9) which in the case of $\Lambda \equiv(\boldsymbol{\alpha}, \boldsymbol{\beta})$ is

$f(\boldsymbol{\alpha}, \boldsymbol{\beta} \mid \boldsymbol{y})=\sum_{\gamma} S t_{p_{\gamma}+p_{0}}\left(\left(\boldsymbol{\alpha}, \boldsymbol{\beta}_{\gamma}\right) \mid\left(\hat{\boldsymbol{\alpha}}, \hat{\boldsymbol{\beta}}_{\gamma}\right),\left(\mathbf{Z}_{\gamma}^{\top} \mathbf{Z}_{\gamma}\right)^{-1} \frac{S S E_{\gamma}}{n-p_{\gamma}-p_{0}}, n-p_{\gamma}-p_{0}\right) \operatorname{Pr}\left(M_{\gamma} \mid \boldsymbol{y}\right)$,

where $\hat{\boldsymbol{\alpha}}, \hat{\boldsymbol{\beta}}_{\gamma}$ is the maximum likelihood estimator under $M_{\gamma}$ (see (4)), $\boldsymbol{Z}_{\gamma}=\left(\boldsymbol{X}_{0}, \boldsymbol{X}_{\gamma}\right)$ and $S S E_{\gamma}$ is the sum of squared errors in $M_{\gamma}$. St above refers to the multivariate student distribution:

$$
S t_{k}(\boldsymbol{x} \mid \boldsymbol{\mu}, \boldsymbol{\Sigma}, d f) \propto\left(1+\frac{1}{d f}(\boldsymbol{x}-\boldsymbol{\mu})^{\top} \boldsymbol{\Sigma}^{-1}(\boldsymbol{x}-\boldsymbol{\mu})\right)^{-(d f+k) / 2} .
$$


In Bayes VarSel the whole model averaged distribution in (10) is provided in the form of a random sample through the function BMAcoeff which depends on two arguments: $\mathrm{x}$, a "Bvs" object and n.sim, the number of observations to be simulated (with default value of 10000). The returned object is an object of class "bma. coeffs" which is a columnnamed matrix with $\mathrm{n}$. sim rows (one per each simulation) and $p+p_{0}$ columns (one per each regression parameter). The way that BMAcoeff works depends on whether the object was created with Bvs or with GibbsBvs. This is further explained below.

If the Bvs object was generated with Bvs In this case the models over which the average is performed are the $\mathrm{n}$. keep (previously specified) best models. Hence, if $\mathrm{n}$. keep equals $2^{p}$ then all competing models are used; while, if $n . k e e p<2^{p}$, only a proportion of them are used and posterior probabilities are re-normalized to sum to one.

On many occasions where estimations are required, the default value of $n$. keep (which we recall is 10) is small and should be increased. Ideally $2^{p}$ should be used but, as noticed by Raftery et al. (1997) this is normally unfeasible and commonly it is sufficient to average over a reduced set of good models that accumulate a reasonable posterior mass. This set is what Raftery et al. (1997) call the "Occam's window." The function BMAcoeff informs about the total probability accumulated in the models that are used.

For illustrative purposes, let us retake the UScrime dataset and, in particular, the example in first section in which, apart from the constant, the variable Ed was assumed as fixed. The total number of models is $2^{14}=16384$ and we execute Bvs again but now with $n$. keep=2000:

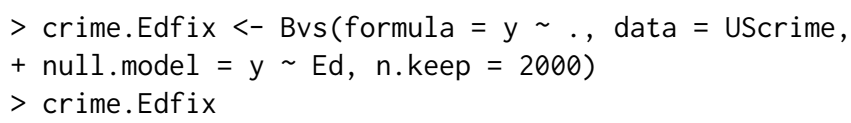

(This takes about 1.9 seconds). The crime. Edfix object contains identical information as the one previously created with the same name, except for the models retained, which in this case are the best 2000 . These models accumulate a probability ${ }^{3}$ of roughly 0.90 , which seems quite reasonable to derive the estimates. We do so executing the second command of the following script (the seed is fixed for the sake of reproducibility).

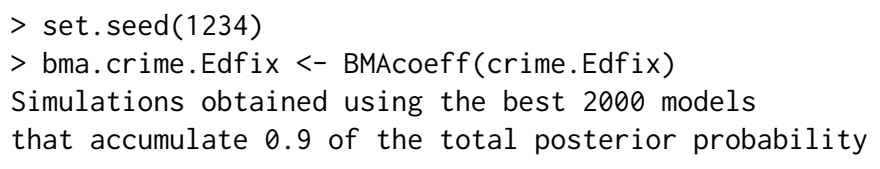

The distribution in (10) and hence the simulations obtained can be highly multimodal and providing default summaries of it (like the mean or standard deviation) is potentially misleading. For a first exploration of the model averaged distribution, BayesVarSel comes armed with a plotting function, histBMA, that produces a histogram-like representation borrowing ideas from Scott and Berger (2006) and placing a bar at zero with height proportional to the number of zeros obtained in the simulation.

The function histBMA depends on several arguments:

- x An object of class bma. coeffs.

- covariate A string specifying the name of an explanatory variable whose accompanying coefficient is to be represented. This must be the name of one of the columns in $\mathrm{x}$.

- n. breaks The number of equal width bars for the histogram. Default value is 100 .

- text If set to TRUE (default value) the frequency of zeroes is added at the top of the bar at zero.

- gray. 0 A numeric value between 0 and 1 that specifies the darkness, in a gray scale ( 0 is white and 1 is black) of the bar at zero. Default value is 0.6 .

- gray. no 0 A numeric value between 0 and 1 that specifies the darkness, in a gray scale ( 0 is white and 1 is black) of the bars different from zero. Default value is 0.8 .

\footnotetext{
${ }^{3}$ sum (crime.Edfix \$modelsprob\$prob)
} 

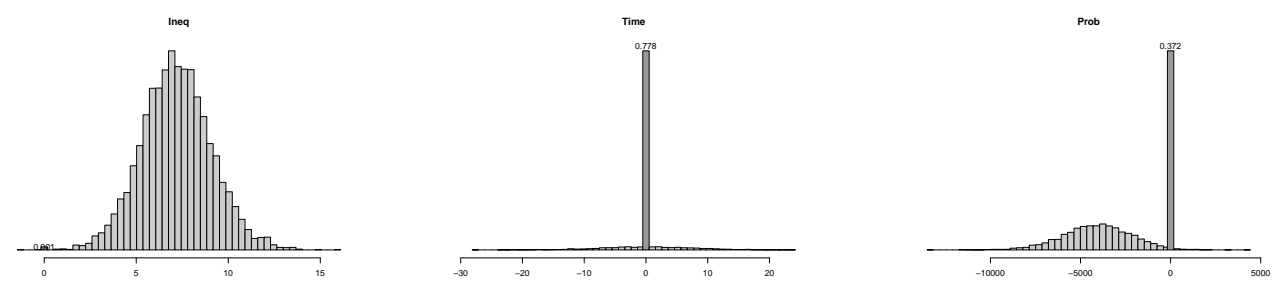

Figure 3: Representation provided by the function histBMA of the Model averaged posterior distributions of $\beta_{\text {Ineq }}, \beta_{\text {Time }}$ and $\beta_{\text {Prob }}$ for the UScrime dataset with a constant and Ed considered as fixed in the variable selection exercise.

For illustrative purposes let us examine the distributions of $\beta_{\text {Ineq }}$ (inclusion probability $0.99) ; \beta_{\text {Time }}(0.24)$ and $\beta_{\text {Prob }}(0.62)$ using histBMA

$>$ histBMA(bma.crime.Edfix, covariate $=$ "Ineq", n.breaks $=50)$
$>$ histBMA(bma.crime.Edfix, covariate $=$ "Time", n.breaks $=50)$
$>$ histBMA(bma.crime.Edfix, covariate $=$ "Prob", n.breaks $=50)$

The plots obtained are reproduced in Figure 3. We can see that Ineq has a positive effect. This distribution is unimodal so there is no drawback to summarizing the effect of the response Ineq over y (crime) using say, the mean or quantiles, For example:

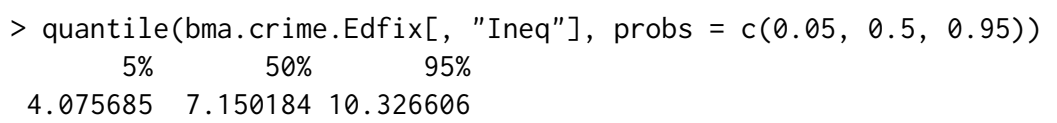

This implies an estimated effect of 7.2 with a $90 \%$ credible interval [4.1,10.3]. The situation of Time is clear and its estimated effect is basically null (in agreement with a low inclusion probability).

Much more problematic is reporting estimates of the effect of Prob with a highly polarized estimated effect being either very negative (around -4100) or zero (again in agreement with its inconclusive inclusion probabilty of 0.62 ). Notice that, in this case, the mean (approximately -2500) should not be used as a sensible estimation of the parameter $\beta_{\text {Prob }}$.

If the Bvs object was generated with GibbsBvs In this case, the average in (10) is performed over the $n$. iter (an argument previously defined) models sampled in the MCMC scheme. Theoretically this corresponds to sampling over the whole distribution (all models are considered) and leads to the approximate method pointed out in Raftery et al. (1997). All previous considerations regarding the difficult nature of the underlying distribution apply here.

Let us consider again the SDM dataset in which analysis we created growth.wstar7 in the fourth section. Suppose we are interested in the effect of the variable P60 on the response GDP. The summary method informs that this variable has an inclusion probability of 0.77 .

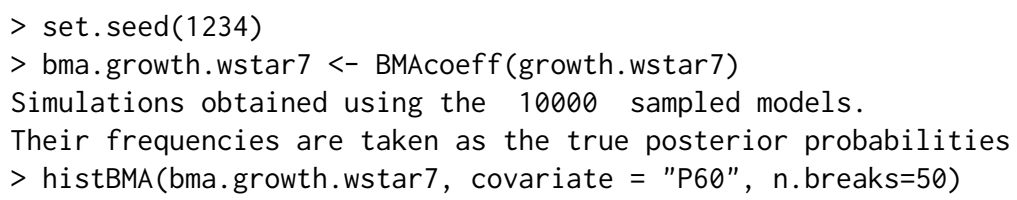

The distribution is bimodal (graph not shown here to save space) with modes at zero and 2.8, approximately. Again, it is difficult to provide simple summaries to describe the model averaged behaviour of P60. Nevertheless, it is always possible to answer relevant questions such as: what is the probability that the effect of P60 over savings is greater than one?

$>$ mean (bma.growth.wstar7[, "P60"] > 1)

[1] 0.7511

\section{Prediction}

Suppose we want to predict a new observation $y^{\star}$ with associated values of covariates $\left(x^{\star}\right)^{\top} \in \mathcal{R}^{p_{0}+p}$ (in what follows, the product of two vectors corresponds to the usual scalar 


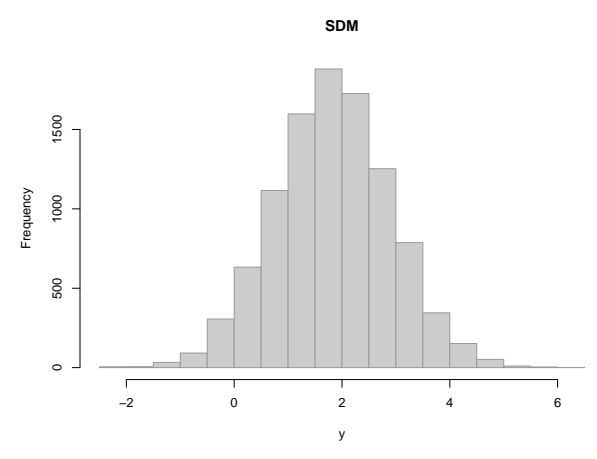

Figure 4: For SDM data and related Bvs object growth.wstar7, model averaged prediction of the "mean" case (predicting the output associated with the mean of observed covariates)

product). In this case, the distribution (9) adopts the form

$$
f\left(y^{\star} \mid \boldsymbol{y}, \boldsymbol{x}^{\star}\right)=\sum_{\gamma} S t\left(y^{\star} \mid \boldsymbol{x}_{\gamma}^{\star}\left(\hat{\boldsymbol{\alpha}}, \hat{\boldsymbol{\beta}}_{\gamma}\right), \frac{S S E_{\gamma}}{h_{\gamma}}, n-p_{\gamma}-p_{0}\right) \operatorname{Pr}\left(M_{\gamma} \mid \boldsymbol{y}\right),
$$

where

$$
h_{\gamma}=1-\boldsymbol{x}_{\gamma}^{\star}\left(\left(\boldsymbol{x}_{\gamma}^{\star}\right)^{\top} \boldsymbol{x}_{\gamma}^{\star}+\mathbf{Z}_{\gamma}^{\top} \mathbf{Z}_{\gamma}\right)^{-1}\left(\boldsymbol{x}_{\gamma}^{\star}\right)^{\top} .
$$

As with estimations, Bayes VarSel has implemented a method for the S3 function predict, designed to simulate a desired number of observations from (11). A main difference with model averaged estimations is that, typically, the above predictive distribution is unimodal.

The predict method depends on object, an object of class "Bvs", newdata, a data frame with the values of the covariates (the intercept, if needed, is automatically added) and n. sim the number of observations to be simulated. The considerations about the calculation of the probabilities described in the previous section for the Bvs object depending on the type of function originally used apply here.

The predict method returns a matrix with $\mathrm{n}$. sim rows (one per each simulated observation) and with the number of columns being the number of cases (rows) in the data frame newdata.

For illustrative purposes, consider the "Bvs" object named growth.wstar7 from the analysis of the SDM dataset. Simulations from the predictive distribution (11) associated with values of the covariates fixed at their means can be obtained with the following code. Here, a histogram is produced (see Figure 4) as a graphical approximation of the underlying distribution.

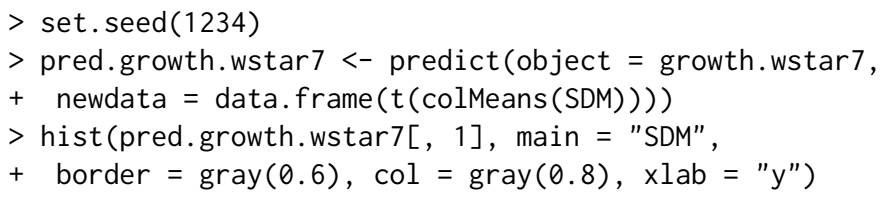

\section{Future work}

The first version of BayesVarSel was released on December 2012 with the main idea of making available the $C$ code programmed for the work Garcia-Donato and Martinez-Beneito (2013) to solve exactly moderate to large variable selection problems. Since then, seven versions have followed with new abilities that make up the complete toolbox that we have described in this paper.

Nevertheless, BayesVarSel is an ongoing project that we plan to continue in the future as solid and contrasted methods become available. The emphasis is placed on the prior distribution that should be used, since this is a particularly relevant aspect of model selection/testing problems.

New functionalities that we expect to incorporate in the future are: 
- The case where $n<p+p_{0}$ and possibly $n<<p+p_{0}$,

- specific methods for handling factors,

- heteroscedastic errors,

- other types of error distributions.

\section{Acknowledgments}

This paper was supported in part by the Spanish Ministry of Economy and Competitivity under grant MTM2016-77501-P (BAiCORRE). The authors would like to thank the referees for their many valuable comments that helped to greatly improve both, this paper and our package BayesVarSel. Also, we would like to thank Jim Berger for his suggestions during the process of building the package and Carlos Vergara for his invaluable help with the use of the versions control system Git.

\section{Bibliography}

M. M. Barbieri and J. O. Berger. Optimal predictive model selection. The Annals of Statistics, 32(3):870-897, 2004. URL https://doi.org/10.1214/009053604000000238. [p164]

M. J. Bayarri and G. García-Donato. Extending conventional priors for testing general hypotheses in linear models. Biometrika, 94(1):135-152, 2007. URL https://doi . org/10. 1093/biomet/asm014. [p159]

M. J. Bayarri, J. O. Berger, A. Forte, and G. García-Donato. Criteria for Bayesian model choice with application to variable selection. The Annals of Statistics, 40:1550-1577, 2012. URL https://doi .org/10.1214/12-A0S1013. [p159,173]

D. A. Belsley, E. Kuh, and R. E. Welsch. Regression Diagnostics: Identifying Influential Data and Sources of Collinearity. John Wiley \& Sons, 2005. URL https://doi.org/10.1002/ 0471725153. [p156]

J. O. Berger. The case for objective Bayesian analysis. Bayesian Analysis, 1(3):385-402, 2006. URL https://doi .org/10.1214/06-BA115. [p155]

J. O. Berger and J. M. Bernardo. On the development of the reference prior method. In J. M. Bernardo, editor, Bayesian Statistics 4, pages 35-60. London: Oxford University Press, 1992. [p167]

J. O. Berger and L. R. Pericchi. Objective Bayesian Methods for Model Selection: Introduction and Comparison. In Model Selection, volume 38 of Lecture Notes-Monograph Series, pages 135-207. Institute of Mathematical Statistics, Beachwood, OH, 2001. URL https://doi. org/10.1214/1nms/1215540968. [p158]

J. O. Berger, J. M. Bernardo, and D. Sun. The formal definition of reference priors. The Annals of Statistics, 37(2):905-938, 2009. URL https://doi .org/10.1214/07-A0S587. [p167]

J. M. Bernardo and A. F. M. Smith. Bayesian Theory. John Wiley \& Sons, 1994. [p167]

M. Clyde. BAS: Bayesian Adaptive Sampling for Bayesian Model Averaging, 2017. URL http: //CRAN. R-project. org/package=BAS. R package version 1.4.7. [p158]

G. Consonni and L. Deldossi. Objective bayesian model discrimination in follow-up experimental designs. Test, 25:397-412, 2016. URL https://doi .org/10.1007/s11749-0150461-3. [p167]

I. Ehrlich. Participation in illegitimate activities: a theoretical and empirical investigation. Journal of Political Economy, 81(3):521-567, 1973. [p161]

J. Faraway. Practical Regression and Anova in R, 2002. [p156, 160] 
J. Faraway. Functions and Datasets for Books by Julian Faraway, 2016. URL http://CRAN. Rproject. org/package=faraway. $\mathrm{R}$ package version 1.0.7. [p156]

C. Fernández, E. Ley, and M. F. Steel. Benchmark priors for Bayesian model averaging. Journal of Econometrics, 100:381-427, 2001. URL https://doi .org/10.1016/S0304$4076(00) 00076-2$. [p174]

A. Forte, G. Garcia-Donato, and M. F. J. Steel. Methods and Tools for Bayesian Variable Selection and Model Averaging in Univariate Linear Regression. International Statistical Review (to appear), 2018. URL https://doi.org/10.1111/insr. 12249. [p158]

G. Garcia-Donato and A. Forte. BayesVarSel: Bayes Factors, Model Choice and Variable Selection In Linear Models, 2015. URL http://CRAN. R-project.org/package=BayesVarSel. R package version 1.6.1. [p155]

G. Garcia-Donato and M. A. Martinez-Beneito. On Sampling Strategies in Bayesian Variable Selection Problems with Large Model Spaces. Journal of the American Statistical Association, 108(501):340-352, 2013. URL https://doi .org/10.1080/01621459.2012.742443. [p163, $164,170]$

E. I. George and R. E. McCulloch. Approaches for Bayesian variable selection. Statistica Sinica, 7:339-373, 1997. [p163]

H. Jeffreys. Theory of Probability. Oxford University Press, 3rd edition, 1961. [p174]

R. E. Kass and A. E. Raftery. Bayes factors. Journal of the American Statistical Association, 90(430):773-795, 1995. URL https://doi . org/10.1080/01621459.1995.10476572. [p156, 158]

R. E. Kass and L. Wasserman. A reference Bayesian test for nested hypotheses and its relationship to the Schwarz criterion. Journal of the American Statistical Association, 90(431): 928-934, 1995. URL https://doi.org/10.1080/01621459.1995.10476592. [p174]

P. M. Lee. Bayesian Statistics. An Introduction. Second Edition. John Wiley \& Sons, 1997. [p155]

E. Ley and M. F. Steel. On the effect of prior assumptions in Bayesian model averaging with applications to growth regression. Journal of Applied Econometrics, 24(4):651-674, 2009. URL https://doi.org/10.1002/jae.1057. [p160,162]

E. Ley and M. F. J. Steel. Jointness in Bayesian variable selection with applications to growth regression. Journal of Macroeconomics, 29(3):476 - 493, 2007. URL https://doi .org/10. $1016 / j$.jmacro.2006.12.002. Special Issue on the Empirics of Growth Nonlinearities. [p161, 165]

F. Liang, R. Paulo, G. Molina, M. A. Clyde, and J. O. Berger. Mixtures of g-priors for Bayesian variable selection. Journal of the American Statistical Association, 103(481):410-423, 2008. URL https://doi .org/10.1198/016214507000001337. [p174]

D. Madigan and A. E. Raftery. Model selection and accounting for model uncertainty in graphical models using Occam's window. Journal of the American Statistical Association, 89: 1535 - 1546, 1994. URL https://doi .org/10.1080/01621459.1994.10476894. [p167]

R. D. Morey, J. N. Rouder, and T. Jamil. BayesFactor: Computation of Bayes Factors for Common Designs, 2015. URL http: //CRAN. R-project.org/package=BayesFactor. R package version 0.9.11-1. [p158]

A. Raftery, J. Hoeting, C. Volinsky, I. Painter, and K. Y. Yeung. BMA: Bayesian Model Averaging, 2015. URL http://CRAN. R-project. org/package=BMA. R package version 3.18.4. [p158]

A. E. Raftery. Bayesian model selection in social research. Sociological Methodology, 25:111 163, 1995. URL http://doi .org/10.2307/271063. [p167]

A. E. Raftery, D. Madigan, and J. Hoeting. Bayesian model averaging for linear regression models. Journal of the American Statistical Association, 92:179-191, 1997. [p168, 169] 
N. Ravishanker and D. K. Dey. A First Course in Linear Model Theory. Chapman and Hall/CRC, 2002. [p159]

D. Rossell, J. D. Cook, D. Telesca, and P. Roebuck. Mombf: Moment and Inverse Moment Bayes Factors, 2014. URL http: //CRAN. R-project. org/package=mombf. R package version 1.5.9. [p158]

X. Sala-I-Martin, G. Doppelhofer, and R. I. Miller. Determinants of long-term growth: A Bayesian averaging of classical estimates (BACE) approach. American Economic Review, 94 (4):813-835, 2004. URL https://doi .org/10.1257/0002828042002570. [p161]

J. G. Scott and J. O. Berger. An exploration of aspects of Bayesian multiple testing. Journal of Statistical Planning and Inference, 136(7):2144-2162, 2006. URL https://doi .org/10.1016/ j.jspi.2005.08.031. [p159,168]

W. N. Venables and B. D. Ripley. Modern Applied Statistics with S. Springer-Verlag, New York, 4th edition, 2002. URL http://www. stats.ox.ac.uk/pub/MASS4. ISBN 0-387-95457-0. [p161]

A. Zellner. On assessing prior distributions and Bayesian regression analysis with g-prior distributions. In A. Zellner, editor, Bayesian Inference and Decision Techniques: Essays in Honor of Bruno De Finetti, pages 389-399. Edward Elgar Publishing Limited, 1986. [p174]

A. Zellner and A. Siow. Posterior odds ratio for selected regression hypotheses. In J. M. Bernardo, M. H. DeGroot, D. V. Lindley, and A. F. M. Smith, editors, Bayesian Statistics 1, pages 585-603. Valencia: University Press, 1980. [p174]

A. Zellner and A. Siow. Basic Issues in Econometrics. Chicago: University of Chicago Press, 1984. [p174]

S. Zeugner and M. Feldkircher. Bayesian model averaging employing fixed and flexible priors: The BMS package for R. Journal of Statistical Software, 68(4):1-37, 2015. URL https://doi.org/10.18637/jss.v068.i04. [p158]

Gonzalo Garcia-Donato

Universidad de Castilla-La Mancha (Department of Economy and Finance; Instituto de Desarrollo Regional (IDR))

Plaza Universidad 2, Albacete

Spain

gonzalo.garciadonato@uclm.es

Anabel Forte

Universidad de Valencia (Department of Statistics and OR) Calle Dr. Moliner, Burjassot (Valencia) Spain

anabel. forte@uv.es

\section{Appendix: Model selection priors for parameters within models}

A key technical component of Bayes factors and hence of posterior probabilities is the prior distribution for the parameters within each model. That is, the prior $\pi_{\gamma}\left(\boldsymbol{\alpha}, \boldsymbol{\beta}_{\gamma}, \sigma\right)$ for the specific parameters of the model

$$
M_{\gamma}: \boldsymbol{y}=\boldsymbol{X}_{0} \boldsymbol{\alpha}+\boldsymbol{X}_{\gamma} \boldsymbol{\beta}_{\gamma}+\varepsilon, \varepsilon \sim N_{n}\left(\mathbf{0}, \sigma^{2} \boldsymbol{I}_{n}\right) .
$$

In Bayes VarSel the prior used is specified in main functions Btest, Bvs, and GibbsBvs with the argument prior. betas with default value "Robust"' that corresponds to the proposal the same name in (Bayarri et al., 2012). It is argued in this paper, based on foundational arguments, that the robust prior is an optimal choice for testing in linear models. 
The robust prior for $M_{\gamma}$ can be specified hierarchically as

$$
\pi_{\gamma}^{R}\left(\boldsymbol{\alpha}, \boldsymbol{\beta}_{\gamma}, \sigma\right)=\sigma^{-1} N_{p_{\gamma}}\left(\boldsymbol{\beta}_{\gamma} \mid \mathbf{0}, g \boldsymbol{\Sigma}_{\gamma}\right),
$$

where $\boldsymbol{\Sigma}_{\gamma}=\sigma^{2}\left(\boldsymbol{V}_{\gamma}^{\top} \boldsymbol{V}_{\gamma}\right)^{-1}$, with

$$
\boldsymbol{V}_{\gamma}=\left(\boldsymbol{I}_{n}-\boldsymbol{X}_{0}\left(\boldsymbol{X}_{0}^{\top} \boldsymbol{X}_{0}\right)^{-1} \boldsymbol{X}_{0}^{\top}\right) \boldsymbol{X}_{\gamma}
$$

and

$$
g \sim p_{\gamma}^{R}(g)=\frac{1}{2} \sqrt{\frac{1+n}{p_{\gamma}+p_{0}}}(g+1)^{-3 / 2}, \quad g>\frac{1+n}{p_{\gamma}+p_{0}}-1 .
$$

For the null model, the prior assumed is $\pi_{0}(\boldsymbol{\alpha}, \sigma)=\sigma^{-1}$.

The idea of using the matrix $\boldsymbol{\Sigma}_{\gamma}$ to scale variable selection priors dates back to Zellner and Siow (1980) and is present in other very popular proposals in the literature. As we next describe, these proposals differ about which distribution should be used for the hyperparameter $g$. Many of these can be implemented in BayesVarSel through the argument prior.betas.

- prior. betas="ZellnerSiow" (Jeffreys (1961); Zellner and Siow $(1980,1984)$ ) corresponds to $g \sim \operatorname{IGa}(1 / 2, n / 2)$ (leading to the very famous proposal of using a Cauchy).

- prior . betas="gZellner" (Zellner (1986); Kass and Wasserman (1995)) corresponds to fixing $g=n$ (leading to the so called Unit Information Prior).

- prior . betas="FLS" (Fernández et al. (2001)) corresponds to fixing $g=\max \left\{n, p^{2}\right\}$.

- prior. betas="Liangetal" (Liang et al. (2008)) corresponds to $g \sim \pi(g) \propto(1+$ $g / n)^{-3 / 2}$. 